\title{
Highly Directed Radiation Pattern From a THz Photonic Transmitter With a Two-Dimensional Rampart Slot Array Antenna
}

\author{
Yu-Ru Huang, Chung-Chiu Kuo, Chiu-Min Chiu, Hung-Pin Chen, Tzeng-Fu Kao, Yi-Chun Chen, \\ An-Shyi Liu, Ruey-Beei Wu, Senior Member, IEEE, Pei-Chin Chiu, Jen-Inn Chyi, Senior Member, IEEE, and \\ Chi-Kuang Sun, Senior Member, IEEE
}

\begin{abstract}
In this study, we investigate the directivity of the terahertz (THz) radiation pattern from a newly designed two-dimensional rampart slot array antenna integrated in an edge-coupled membrane photonic transmitter. The antenna design is based on the array theory which is well-developed in the microwave regime. By means of the array arrangement of rampart slot antennas, we demonstrate that the 3-dB beam width of the $\mathrm{THz}$ radiation pattern at $907 \mathrm{GHz}$ is successfully confined within $30^{\circ}$ in both $H$ and $E$ planes.
\end{abstract}

Index Terms-Antenna, array, radiation pattern, terahertz (THz) photonic transmitter.

\section{INTRODUCTION}

W ITH the burgeoning terahertz $(\mathrm{THz})$ wave applications such as molecule imaging system [1] and biodetection technology [2], how to collect $\mathrm{THz}$ waves more efficiently is a key point to design a $\mathrm{THz}$ wave source. We have previously demonstrated an edge-coupled photonic transmitter based on a low-temperature-grown GaAs metal-semiconductor-metal traveling-wave photodectector (MSM-TWPD) and a coplanar-waveguide (CPW)-fed slot dipole antenna. The compact $\mathrm{THz}$ source exhibited not only the wide frequency tunability but also the high conversion efficiency [3]. However, it is well known that the directivity of the radiation pattern, especially in the $E$ plane, from the CPW-fed slot dipole antennas is low [4]. Therefore, the divergent $\mathrm{THz}$

Manuscript received November 13, 2007. This work was supported by the National Science Council through NSC 96-2628-E-002-043-MY3 and NSC 96-2120-M-002-014 and by the Division of Genomic Medicine, NTU Center for Medical Excellence.

Y.-R. Huang, C.-C. Kuo, C.-M. Chiu, H.-P. Chen, and T.-F. Kao are with the Department of Electrical Engineering and Graduate Institute of Photonics and Optoelectronics, National Taiwan University, Taipei 106, Taiwan, R.O.C.

Y.-C. Chen, A.-S. Liu, and R.-B. Wu are with the Department of Electrical Engineering and Graduate Institute of Communication Engineering, National Taiwan University, Taipei 106, Taiwan, R.O.C.

P.-C. Chiu and J.-I. Chyi are with the Optical Sciences Center and Department of Electrical Engineering, Nation Central University, Taoyuan 320, Taiwan, R.O.C.

C.-K. Sun is with the Department of Electrical Engineering and Graduate Institute of Photonics and Optoelectronics, National Taiwan University and Research Center for Applied Sciences, Academia Sinica, Taipei 106, Taiwan, R.O.C.

Color versions of one or more of the figures in this letter are available online at http://ieeexplore.ieee.org.

Digital Object Identifier 10.1109/LPT.2008.924197 wave is not so appropriate for some far-field applications such as direct coupling to $\mathrm{THz}$ fibers without high numerical aperture focusing medium.

Many groups had been devoted to developing highly directed $\mathrm{THz}$ radiation sources with the array design such as photoconductive photomixer arrays [5] and photodiode-integrated antenna arrays [6]. However, multiple excitation sources are needed to drive each element in the array and this would make the system too complex for use. Arranging multiple devices in the array also makes the final circuit too large (usually several square millimeters to square centimeters) to be integrated with other circuits or devices.

To realize a compact highly directed $\mathrm{THz}$ wave source, here we demonstrate a new design for the $\mathrm{THz}$ photonic transmitter. A newly designed two-dimensional (2-D) rampart slot array antenna is adopted to centralize the main beam of the radiated $\mathrm{THz}$ waves. Combined with an edge-coupled MSM-TWPD, an ultrahigh resonant frequency at $907 \mathrm{GHz}$ could be observed. Under the coherently controlled optical beating excitation, the measured radiation pattern at $907 \mathrm{GHz}$ is successfully concentrated to less than $30^{\circ}$ in both the $H$ and $E$ planes, which is much smaller than the theoretical limit of a single CPW-fed slot dipole antenna [4]. By integrating the array antennas with a single photodetector, a highly directed $\mathrm{THz}$ wave emitter could be realized rather than combining multiple devices. The fabricated device size is less than $1 \mathrm{~mm}^{2}$, with a high capability for future integration in the optoelectronic integrated circuits and biomicrochips [2].

\section{Design AND StRucture}

The top micrographic view of the fabricated $\mathrm{THz}$ photonic transmitter is shown in Fig. 1. It is composed of an edge-coupled MSM-TWPD, a low-pass filter, a dc probe pad, and a newly designed array antenna. We adopt the MSM-TWPD as our photodetector due to its high-power bandwidth product [7] and superior microwave properties such as higher propagation velocity and lower high-frequency microwave loss [8]-[10]. The lowpass filter was designed by using a THz-wave photonic bandgap structure to reflect the THz-frequency ac currents back to the antenna to enhance the radiation efficiency. A detailed structure is shown in the inset of Fig. 1. The antenna was designed based on the array theory which is well-developed in the microwave regime. Since the excited $\mathrm{THz}$ signal is fed from the CPW after the TWPD, the design of our array antenna can be 


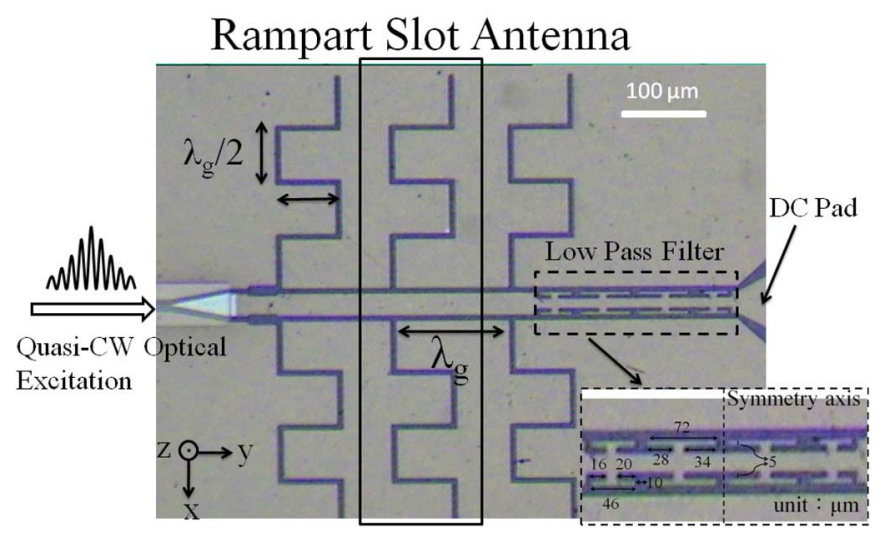

Fig. 1. Top micrographic view of the fabricated membrane photonic transmitter. The left-hand arrow indicates the direction of the coherently controlled quasi-CW optical excitation. The inset shows the detailed structure of the lowpass filter.

simplified without considering the excitation mechanism. As shown in Fig. 1, we arranged three CPW-fed rampart slot antennas in series with an interval of one guided wavelength $\left(\lambda_{g}=\right.$ $156 \mu \mathrm{m})$. The rampart slot array antenna was adopted due to several advantages including easy control of input impedance, small cross polarization level, and high gain [11]. Our rampart slotline has a 7- $\mu \mathrm{m}$ width for input matching while the length of both the vertical and horizontal segments equals to one half of the guided wavelength. Due to the magnetic current distribution in the slotlines, the far-field radiation pattern is only contributed from the vertical segments [11]. Thus, a linear array along the $\mathrm{x}$-axis can be easily achieved by using a single rampart slot antenna and a more confined pattern in the $x-z$ plane ( $H$ plane) can be expected. However, the radiation pattern in the $y-z$ plane ( $E$ plane) from a single rampart slot antenna is still quite broad. To centralize the divergent radiation pattern in the $y-z$ plane $(E$ plane), we arranged multiple rampart slot array antennas along the $y$-axis and separated them with an interval of one guided wavelength. Thus, a linear array in the other dimension could be achieved. Nevertheless, the antenna efficiency would saturate by adding too many elements in the $\mathrm{x}$ - or $\mathrm{y}$-directions [11]. Considering a reasonable device size without sacrificing the efficiency, we choose three rampart pairs in the waveguide direction and eight radiating elements along rampart slotline pairs. We used a 3-D full-wave simulation tool, HFSS, to calculate the return loss and the radiation patterns of our array antennas. As shown in Fig. 2(a), our design had a good impedance match at $910 \mathrm{GHz}$. The corresponding radiation patterns in the $E$ and $H$ planes were shown in Fig. 2(b) and (c). The 3-dB beam width could be confined to $\sim 26^{\circ}$ in the $E$ plane and $\sim 21^{\circ}$ in the $H$ plane. Compared with the traditional CPW-fed slot dipole antenna [4], our new design attains higher directivity in both $E$ and $H$ planes. After combining all the designed elements, the size of the photonic transmitter is about $0.8 \mathrm{~mm}^{2}$.

\section{EXPERIMENTAL SETUP AND RESULTS}

We used a $800-\mathrm{nm}$ femtosecond Ti: sapphire laser, with a 130 -fs pulsewidth and a $82-\mathrm{MHz}$ repetition rate, and a coherent control system to mimic a quasi-continuous-wave excitation [12], [13]. Before being guided into the Michelson interfer-



(a)

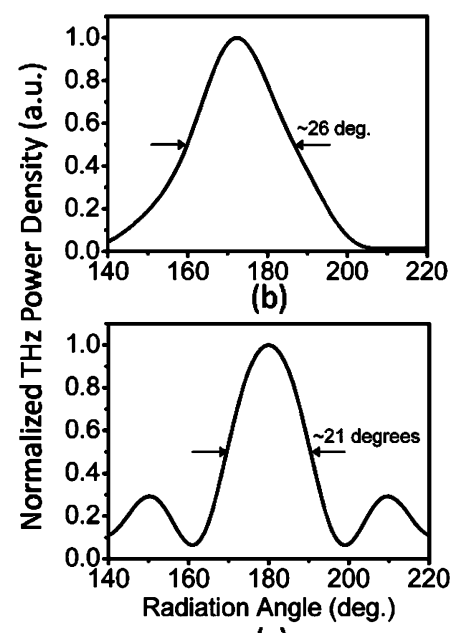

(c)
Fig. 2. (a) Simulated return loss of the 2-D array antenna. The impedance matching point is designed at $910 \mathrm{GHz}$. The simulated radiation patterns in the $E$ and $H$ plane at $910 \mathrm{GHz}$ are shown in (b) and (c). The designed $3-\mathrm{dB}$ beam width is about $26^{\circ}$ and $21^{\circ}$, respectively.

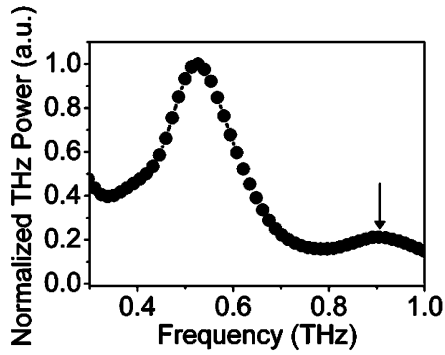

(a)

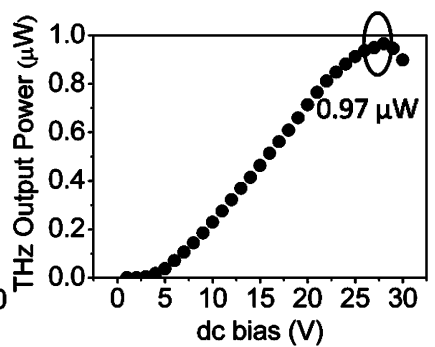

(b)
Fig. 3. (a) Measured frequency response of the THz photonic transmitter. A relatively higher radiation power was observed at $907 \mathrm{GHz}$. (b) Bias dependence of the $\mathrm{THz}$ output power at $907 \mathrm{GHz}$. A $0.97-\mu \mathrm{W}$ output power could be achieved under $4.5-\mathrm{mW}$ optical excitation power and $28-\mathrm{V}$ dc bias.

ometer, the femtosecond laser pulses were passed through a grating pair to be stretched in space and time according to their frequency. After precisely controlling the delay time of the two arms in a Michelson interferometer, we could control the overlap of two stretched pulses in the time domain and thus controlled the optical beating frequency of our excitation. The tuning range of our excitation frequency was up to $1.1 \mathrm{THz}$ [3]. We focused the coherently controlled optical pulses into the absorption region of the photonic transmitter by a microscope objective. After excitation, we used two 3-in parabolic mirrors to collect the radiated $\mathrm{THz}$ wave and focused it into a liquid-He-cooled $\mathrm{Si}$ bolometer. The responsivity of the Si bolometer was calibrated with a method reported before [14]. We inserted a chopper in the optical path and measured the output of the bolometer with a lock-in amplifier. At a $\mathrm{dc}$ bias of $7 \mathrm{~V}$ and under $0.9-\mathrm{mW}$ optical excitation power, the beating-frequency-dependent $\mathrm{THz}$ radiation power was measured and shown in Fig. 3(a). We could observe that there was a relatively higher radiation power at $907 \mathrm{GHz}$, which corresponds to our designed resonant frequency, within the $800-$ to $1000-\mathrm{GHz}$ range. In addition, a strong resonant frequency at about $500 \mathrm{GHz}$ was also observed and could be 




(a)

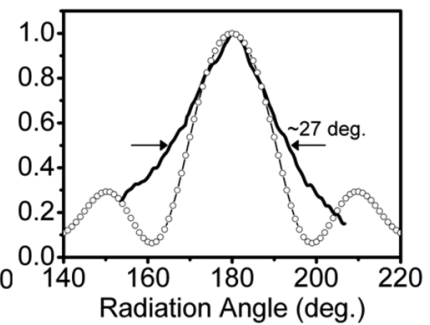

(b)
Fig. 4. Measured radiation patterns (solid lines) at $907 \mathrm{GHz}$ in the (a) $E$ plane and (b) $H$ plane. The simulated radiation patterns (open circles) in both planes at $910 \mathrm{GHz}$ are also plotted for comparison.

attributed to the impedance mismatch at the $2 \lambda$ resonant point [3]. The bias-dependent output power at $907 \mathrm{GHz}$ under a fixed 4.5-mW optical excitation power was also measured. As shown in Fig. 3(b), a maximum average output power of $0.97 \mu \mathrm{W}$ (corresponding to 1.3-mW peak power and 9-ps pulsewidth) could be achieved at a dc bias of $28 \mathrm{~V}$. The corresponding external (including coupling and collection loss) power conversion efficiency is $0.97 \mu \mathrm{W} / 4.5 \mathrm{~mW}=2.14 \times 10^{-4}$, which agrees well with our previous results [3], [14], [15].

The $\mathrm{THz}$ radiation pattern was measured by a knife-cut method. A sharp metal plate was slid into the space between the photonic transmitter and the first collection mirror and its position was controlled by a stepping motor. Under a fixed optical excitation power of $0.9 \mathrm{~mW}$ and a dc bias of $7 \mathrm{~V}$, the measured radiation patterns in the $E$ and $H$ plane at $907 \mathrm{GHz}$ are shown in Fig. 4(a) and (b), respectively. The measured 3-dB beam width was successfully concentrated to $\sim 29^{\circ}$ and $\sim 27^{\circ}$ in the $E$ and $H$ planes. The simulated patterns at $910 \mathrm{GHz}$ (open circles) are also plotted for comparison. Despite the finite collecting angle of the parabolic mirror, which was $\sim 90^{\circ}$ and $\sim 52^{\circ}$ in the $E$ and $H$ plane, respectively, most of the radiated $\mathrm{THz}$ waves could be collected. Therefore, the collection efficiency can be greatly enhanced by the present design. With the aid of the antenna array design, it becomes much easier to centralize and direct the $\mathrm{THz}$ output power to the expected region.

\section{CONCLUSION}

We have demonstrated a novel $\mathrm{THz}$ photonic transmitter which is based on an MSM-TWPD and a CPW-fed 2-D rampart slot antenna array. A centralized $\mathrm{THz}$ radiation pattern in both $H$ and $E$ planes could be achieved without combining multiple $\mathrm{THz}$ sources. Our result shows the potential capability for radiation angle control and will be important for future applications requiring a more confined radiation pattern, such as direct coupling to the $\mathrm{THz}$ fibers and some biodetection devices. The compact size $\left(<1 \mathrm{~mm}^{2}\right)$ is also an advantageous feature for future integration with other active devices.

\section{ACKNOWLEDGMENT}

The authors would like to thank Prof. C.-L. Pan for technical support.

\section{REFERENCES}

[1] J.-Y. Lu, H.-H. Chang, L.-J. Chen, M.-J. Tien, and C.-K. Sun, "Optoelectronic-based high-efficiency quasi-CW terahertz imaging," IEEE Photon. Technol. Lett., vol. 17, no. 11, pp. 2406-2408, Nov. 2005.

[2] J.-Y. Lu, L.-J. Chen, T.-F. Kao, H.-H. Chang, H.-W. Chen, A.-S. Liu, Y.-C. Yu, R.-B. Wu, W.-S. Liu, J.-I. Chyi, and C.-K. Sun, "Terahertz microchip for illicit drug detection," IEEE Photon. Technol. Lett., vol. 18, no. 21, pp. 2254-2256, Nov. 1, 2006.

[3] T.-F. Kao, H.-H. Chang, L.-J. Chen, J.-Y. Lu, A.-S. Liu, Y.-C. Yu, R.-B. Wu, and C.-K. Sun, "Frequency tunability of terahertz photonic transmitters," Appl. Phys. Lett., vol. 88, p. 093501, 2006.

[4] H.-C. Liu, T.-S. Horng, and N. G. Alexopoulos, "Radiation of printed antennas with a coplanar waveguide feed," IEEE Trans. Antennas Propag., vol. 43, no. 10, pp. 1143-1148, Oct. 1995.

[5] D. Saeedkia, A. H. Majedi, S. Safavi-Naeini, and R. P. Mansour, "Analysis and design of a photoconductive integrated photomixer/antenna for terahertz," IEEE J. Quantum Electron., vol. 41, no. 2, pp. 234-241, Feb. 2005.

[6] N. Shimizu and T. Nagatsuma, "Photodiode-integrated microstrip antenna array for subterahertz radiation," IEEE Photon. Technol. Lett., vol. 18, no. 6, pp. 743-745, Mar. 15, 2006.

[7] J.-W. Shi, K.-G. Gan, Y.-H. Chen, C.-K. Sun, Y.-J. Chiu, and J. E. Bowers, "Ultrahigh-power-bandwidth product and nonlinear photoconductance performances of low-temperature-grown GaAs-based metal-semiconductor-metal traveling-wave photodetectors," IEEE Photon. Technol. Lett., vol. 14, no. 11, pp. 1587-1589, Nov. 2002.

[8] J.-W. Shi, K.-G. Gan, Y.-J. Chiu, Y.-H. Chen, C.-K. Sun, Y.-J. Yang, and J. E. Bowers, "Metal-semiconductor-metal traveling-wave photodetectors," IEEE Photon. Technol. Lett., vol. 13, no. 6, pp. 623-625, Jun. 2001.

[9] J.-W. Shi and C.-K. Sun, "Design and analysis of long absorptionlength traveling-wave photodetectors," J. Lightw. Technol., vol. 18, no. 12, pp. 2176-2187, Dec. 2000.

[10] J.-W. Shi, "Metal-semiconductor-metal traveling-wave photodetector," $\mathrm{Ph} . \mathrm{D}$. thesis, Institute of Electro-Optical Engineering, National Taiwan University, Taiwan, R.O.C.

[11] S.-Y. Chen and P. Hsu, "Rampart slot array fed by coplanar waveguide," in Proc. 2002 Asia-Pacific Microwave Conf., Kyoto, Japan, vol. 2 , pp. $1286-1287$.

[12] A. S. Weling and D. H. Auston, "Novel sources and detectors for coherent tunable narrowband terahertz radiation in free space," J. Opt. Soc. Amer. B, vol. 13, pp. 2783-2791, 1996.

[13] L.-J. Chen, T.-F. Kao, J.-Y. Lu, and C.-K. Sun, "A simple terahertz spectrometer based on a low-reflectivity Fabry-Perot interferometer using Fourier transform spectroscopy," Opt. Express, vol. 14, pp. 3840-3846, 2006.

[14] J.-W. Shi, S.-W. Chu, M.-C. Tien, C.-K. Sun, Y.-J. Chiu, and J. E. Bowers, "Edge-coupled membrane terahertz photonic transmitters based on metal-semiconductor-metal traveling-wave photodetectors," Appl. Phys. Lett., vol. 81, pp. 5108-5110, 2002.

[15] M.-C. Tien, H.-H. Chang, J.-Y. Lu, L.-J. Chen, S.-Y. Chen, R.-B. Wu, W.-S. Liu, J.-I. Chyi, and C.-K. Sun, "Device saturation behavior of submillimeter-wave membrane photonic transmitters," IEEE Photon. Technol. Lett., vol. 16, no. 3, pp. 873-875, Mar. 2004. 